\title{
A high-risk phenotype associates with reduced improvement in glycaemia during a lifestyle intervention in prediabetes
}

\author{
Norbert Stefan ${ }^{1,2,3} \cdot$ Harald Staiger ${ }^{1,2,3} \cdot$ Robert Wagner $^{1,2,3}$ - Jürgen Machann ${ }^{2,3,4}$. \\ Fritz Schick $^{2,3,4} \cdot$ Hans-Ulrich Häring ${ }^{1,2,3} \cdot$ Andreas Fritsche $^{1,2,3}$
}

Received: 20 July 2015 / Accepted: 28 August 2015 / Published online: 24 September 2015

(C) Springer-Verlag Berlin Heidelberg 2015

\begin{abstract}
Aims/hypothesis Lack of reversal of prediabetes (impaired glucose tolerance and/or impaired fasting glucose) to normal glucose regulation (NGR) during a lifestyle intervention is strongly associated with a higher incidence of diabetes later in life. In the Tübingen Lifestyle Intervention Program (TULIP) we hypothesised that an at-risk phenotype may exist at baseline that associates with this nonresponse to the intervention.

Methods A total of 120 participants of TULIP with prediabetes at baseline were studied. Participants underwent 9 months of lifestyle intervention and had measurements of insulin secretion and insulin sensitivity during a $75 \mathrm{~g}$ OGTT, and measurements of liver fat content by proton magnetic resonance spectroscopy.

Results During the lifestyle intervention, 55\% of the participants did not revert to NGR. Even among participants with the largest body fat loss (upper quartile: $-6.9 \pm 3.3 \%$, mean $\pm \mathrm{SD}$ ),
\end{abstract}

Electronic supplementary material The online version of this article (doi:10.1007/s00125-015-3760-z) contains peer-reviewed but unedited supplementary material, which is available to authorised users.

Norbert Stefan

norbert.stefan@med.uni-tuebingen.de

1 Department of Internal Medicine IV, Division of Endocrinology, Diabetology, Vascular Medicine, Nephrology and Clinical Chemistry, University Hospital Tübingen, Otfried-Müller-Str. 10, 72076 Tübingen, Germany

2 Institute of Diabetes Research and Metabolic Diseases (IDM) of the Helmholtz Center Munich at the University of Tübingen, Tübingen, Germany

3 German Center for Diabetes Research (DZD), Tübingen, Germany

4 Section of Experimental Radiology, University Hospital Tübingen, Tübingen, Germany
$40 \%$ did not revert to NGR. In this regard, we identified at baseline a high-risk phenotype $(n=72)$ consisting of low disposition index or low insulin sensitivity + nonalcoholic fatty liver disease (NAFLD) and a low-risk phenotype $(n=48$, all other traits). While the adjusted decrease in body fat was almost identical between these phenotypes $(-5.7 \pm 15.3 \%$ vs $-7.7 \pm 15.2 \%, p=0.49)$, the high-risk phenotype had a smaller decrease in adjusted $2 \mathrm{~h}$ blood glucose levels $(-3.7 \pm 20.3 \%$ vs $-18.5 \pm 20.0 \%, p=0.0009)$. In addition, only $31 \%$ of the participants with the high-risk phenotype, but $67 \%$ with the lowrisk phenotype, reverted to NGR $(p<0.0001)$. The odds ratio for reaching the status NGR was $4.54(95 \%$ CI $2.08,9.94)$ for participants having the low-risk phenotype.

Conclusions/interpretation Stratification of individuals with prediabetes at baseline into a high-risk and a low-risk phenotype, based on corrected insulin secretion and insulin-resistant NAFLD, may help to determine the effectiveness of a lifestyle intervention to revert individuals to NGR.

Keywords Impaired glucose tolerance $\cdot$ Insulin secretion · Insulin sensitivity $\cdot$ Lifestyle intervention $\cdot$ Nonalcoholic fatty liver disease $\cdot$ Normal glucose regulation $\cdot$ Prediabetes

$\begin{array}{ll}\text { Abbreviations } \\ \text { BIA } & \text { Bioelectrical impedance } \\ \text { D }_{\text {I }} & \text { Disposition index } \\ \text { DPS } & \text { Diabetes Prevention Study } \\ \text { DPP } & \text { Diabetes Prevention Program } \\ \text { DPPOS } & \text { Diabetes Prevention Program Outcome Study } \\ { }^{1} \text { H-MR } & \text { Proton magnetic resonance } \\ \text { HPA } & \text { Habitual physical activity } \\ \text { IFG } & \text { Impaired fasting glucose } \\ \text { IGT } & \text { Impaired glucose tolerance } \\ \text { MRT } & \text { Magnetic resonance tomography }\end{array}$


NAFLD Nonalcoholic fatty liver disease

NFG Normal fasting glucose

NGT Normal glucose tolerance

NGR Normal glucose regulation

TULIP Tübingen Lifestyle Intervention Program

\section{Introduction}

In several clinical trials, lifestyle intervention with modification of diet and an increase in physical activity has prevented type 2 diabetes in people with prediabetes (impaired glucose tolerance $[\mathrm{IGT}]$ and/or impaired fasting glucose [IFG]) [1-10]. However, although this safe intervention is generally very effective at preventing diabetes, some people still develop the disease $[1,3]$. Thus, it is important to understand which phenotypic and genetic variables are able to determine the effectiveness of a lifestyle intervention to prevent diabetes. In this respect, in the Finnish Diabetes Prevention Study (DPS), age and initial diabetes risk (estimated from the Finnish Diabetes Risk Score), but not estimated body fat mass and body fat distribution or insulin sensitivity (estimated from fasting glucose and insulin levels), were associated with the effectiveness of the lifestyle intervention to prevent diabetes [11].

The prevention of incident diabetes in prediabetes is a commonly used endpoint to study the effectiveness of an intervention. However, for the individual participant it may take several years until such an intervention proves to be effective or not. Thus, it would be desirable to have an intermediate endpoint that can be investigated after a short period of time during the intervention, and which proved to strongly predict incident diabetes later on in life. In this respect, Perreault et al showed in the U.S. Diabetes Prevention Program Outcome Study (DPPOS) that specifically the reversal of prediabetes and the restoration of normal glucose regulation (NGR) during intensive lifestyle intervention were strongly associated with a reduced incidence of diabetes [12]. Perreault et al also identified lower fasting and $2 \mathrm{~h}$ blood glucose levels, younger age and higher insulin secretion as baseline variables predicting a higher chance for regression towards NGR in all three treatment groups combined [13].

While this study elegantly provided information that the continuous variables glycaemia, insulin secretion and age associate with the effectiveness of an intervention to reach the level of NGR, in routine clinical practice it is necessary to have categories of metabolic traits, so-called phenotypes, which enable the categorisation of patients into the respective high- and low-risk groups. Therefore, in the present study we specifically investigated whether using pathophysiologybased traits such as low insulin secretion, low insulin sensitivity, nonalcoholic fatty liver disease (NAFLD), visceral adiposity and others may enable the identification of a unique at-risk phenotype that associates with the nonresponse to a structured lifestyle intervention in respect to restoration of NGR.

\section{Methods}

We analysed data from the German Tübingen Lifestyle Intervention Program (TULIP) [14-16]. In this study, white individuals were included when they fulfilled at least one of the following criteria: a family history of type 2 diabetes (ascertained by questionnaires and by interviews with trained staff; defined as having at least one first degree relative with type 2 diabetes); a BMI $>27 \mathrm{~kg} / \mathrm{m}^{2}$; or a diagnosis of IGT and/ or of previous gestational diabetes. A total of 120 participants had IFG [fasting glucose $\geq 5.6 \mathrm{mmol} / \mathrm{l}(\geq 100 \mathrm{mg} / \mathrm{dl})$ ] and/or IGT $[2 \mathrm{~h}$ glucose $\geq 7.8 \mathrm{mmol} / 1(\geq 140 \mathrm{mg} / \mathrm{dl})]$ and measurements of liver fat content at baseline. None of these participants was on any glucose-lowering medication, $28 \%$ were on antihypertensive and $12 \%$ were on lipid-lowering medication. Informed written consent was obtained from all participants and the local medical ethics committee had approved the protocol.

Lifestyle intervention After the baseline measurements, individuals underwent dietary counselling and had up to ten sessions with a dietitian. Participants presented a 3 day food intake protocol at each visit. By individual counselling, the aim was to reduce the intake of fat to amounts lower than $30 \%$ of total energy intake, to reduce the intake of saturated fat to amounts lower than $10 \%$ of total fat intake, to increase the intake of fibre to $15 \mathrm{~g} / 4,185 \mathrm{~kJ}(1,000 \mathrm{kcal})$ and to achieve a weight loss $>5 \%$ during the study. Diet composition was estimated with a validated computer program using two representative days of a 3 day diary (DGE-PC 3.0; Deutsche Gesellschaft für Ernährung, Bonn, Germany). Individuals were asked to perform at least $3 \mathrm{~h}$ of moderate sports per week. Aerobic endurance exercise (e.g. walking, swimming) with only moderate increase in the heart rate was encouraged. To determine the optimal aerobic exercise intensity, participants performed an incremental exercise test on a motorised treadmill (Saturn; HP-Cosmos, Traunstein, Germany) using a walking protocol and the heart rate at their individual lactate threshold was determined [17]. Individuals were given a heart rate monitor (Polar, Büttelborn, Germany) to measure their heart rate during exercise and it was recommended that they exercise with a heart rate close to, but below, the heart rate at their individual lactate threshold. The anthropometric, metabolic, physical activity and energy intake measurements were repeated after 9 months.

Habitual physical activity All individuals completed a standardised self-administered and validated questionnaire to 
measure physical activity, and a habitual physical activity (HPA) score was calculated [18].

Total body fat, body fat distribution, lean body mass and liver fat content Measurements of total body fat and visceral fat and lean body mass were performed by an axial T1weighted fast spin echo technique with a $1.5 \mathrm{~T}$ whole-body magnetic resonance imager. Liver fat content was measured by localised proton magnetic resonance $\left({ }^{1} \mathrm{H}-\mathrm{MR}\right)$ spectroscopy as previously described [19]. NAFLD was defined as liver fat content $>5.56 \%$ [20]. Magnetic resonance tomography (MRT) and ${ }^{1} \mathrm{H}-\mathrm{MR}$ spectroscopy measurements were available in all participants at baseline, but only in 103 and 101 participants at follow-up. However, total body fat measurements by the tetrapolar bioelectrical impedance (BIA) method (expressed in \% body weight) were available in all participants at baseline and at follow-up.

Oral glucose tolerance test and clinical chemical analyses Participants underwent a frequently sampled $2 \mathrm{~h}, 75 \mathrm{~g}$ OGTT. Venous plasma samples were obtained at $0,30,60,90$ and 120 min for determination of plasma glucose and insulin levels. Blood glucose was determined using a bedside glucose analyser (YSI, Yellow Springs, CO, USA). Plasma insulin was determined on an ADVIA Centaur XP and all other routine variables on an ADVIA 1800 clinical chemistry system (Siemens Healthcare systems, Erlangen, Germany). NGR (fasting glucose $<5.6 \mathrm{mmol} / 1$ and $2 \mathrm{~h}$ glucose $<7.8 \mathrm{mmol} / \mathrm{l}$ ), prediabetes and diabetes were diagnosed according to the recommendations of the 2003 International Expert Committee report on the diagnosis of diabetes [21]. Whole-body insulin sensitivity was calculated from glucose and insulin values during the OGTT as proposed by Matsuda and DeFronzo [22]. Insulin secretion was determined by calculation of the insulinogenic index (insulin at $30 \mathrm{~min}-$ insulin at $0 \mathrm{~min}$ )/(glucose at $30 \mathrm{~min}-$ glucose at $0 \mathrm{~min})$. The disposition index $\left(\mathrm{D}_{\mathrm{I}}\right)$, an estimate of beta cell function relative to insulin sensitivity, was calculated as the product of insulin sensitivity and insulin secretion.

Statistical analyses Data are given as means \pm SD. For statistical analyses, data that were not normally distributed
(Shapiro-Wilk $W$ Test, e.g. age, insulin sensitivity, insulinogenic index, $\mathrm{D}_{\mathrm{I}}$, liver fat content, fat mass, fat distribution) were logarithmically transformed to approximate a normal distribution. Pearson correlations and the Student's $t$ test were used to investigate univariate relationships. To test which baseline variables best associate with the improvement in glycaemia during the intervention we focused on sex, age, BMI, waist circumference, baseline fasting and $2 \mathrm{~h}$ glucose levels, insulin secretion, insulin sensitivity, liver fat content and insulin sensitivity $\times$ liver fat content. The variables were chosen because they were found to be associated with the improvement of glucose tolerance during lifestyle intervention $[13,23,24]$. The variable insulin sensitivity $\times$ liver fat content was included based on the existence of a metabolically benign and malignant NAFLD [25]. On the basis of these findings, high- and low-risk phenotypes were defined. Differences in anthropometrics and metabolic variables between the high- and low-risk groups in the cross-sectional analysis were tested using multivariate linear regression analysis with sex, age and body fat as covariates. The high- and low-risk groups were included in the models as independent variables. Changes in body weight, body fat and glucose levels between baseline and 9 months of follow-up during the lifestyle intervention were also tested in multivariate linear regression analysis. Here fold-changes (follow-up value/baseline value) in body weight, body fat and fasting and $2 \mathrm{~h}$ glucose levels were the dependent variables, while age, sex and the respective level of the variable at baseline were the independent variables. A $p$ value $\leq 0.05$ was considered statistically significant.

\section{Results}

Cross-sectional results In our participants with prediabetes at baseline (electronic supplementary material [ESM] Table 1), insulin sensitivity correlated with body fat measured by BIA and MRT, as well as with visceral fat mass and liver fat content. These relationships were weaker or absent between $\mathrm{D}_{\mathrm{I}}$, fasting and $2 \mathrm{~h}$ glucose levels and these variables (Table 1). Compared with individuals without NAFLD, individuals with NAFLD had lower insulin sensitivity $(7.3 \pm 3.7$ vs $12.1 \pm$ $4.9 \mathrm{AU}, p<0.0001)$ and higher fasting $(5.8 \pm 0.7$ vs $5.6 \pm$
Table 1 Relationships of body fat mass and body fat distribution with metabolic variables at baseline

\begin{tabular}{|c|c|c|c|c|c|c|c|c|}
\hline \multirow[t]{2}{*}{ Variable } & \multicolumn{2}{|c|}{ Body fat ${ }_{\text {BIA }}$} & \multicolumn{2}{|c|}{ Body fat $_{\text {MRT }}$} & \multicolumn{2}{|c|}{ Visceral fat ${ }_{M R T}$} & \multicolumn{2}{|c|}{ Liver fat $_{\text {MRS }}$} \\
\hline & $r$ & $p$ & $r$ & $p$ & $r$ & $p$ & $r$ & $p$ \\
\hline Insulin sensitivity & -0.29 & 0.001 & -0.43 & $<0.0001$ & -0.49 & $<0.0001$ & -0.57 & $<0.0001$ \\
\hline $\mathrm{D}_{\mathrm{I}}$ & -0.18 & 0.06 & -0.14 & 0.14 & -0.20 & 0.03 & -0.18 & 0.047 \\
\hline Fasting glucose & -0.04 & 0.63 & 0.03 & 0.74 & 0.29 & 0.001 & 0.15 & 0.11 \\
\hline $2 \mathrm{~h}$ glucose & 0.19 & 0.03 & 0.16 & 0.08 & 0.16 & 0.09 & 0.27 & 0.003 \\
\hline
\end{tabular}

$r$, Pearson correlation coefficient; MRS, magnetic resonance spectroscopy 


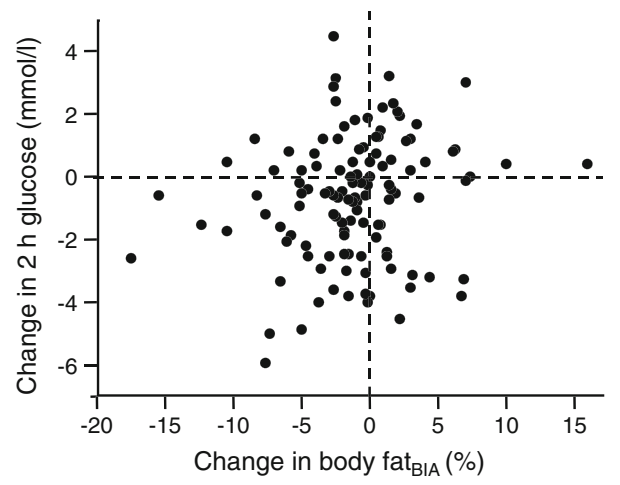

Fig. 1 Relationship between changes in body fat and changes in $2 \mathrm{~h}$ glucose levels during lifestyle intervention. Data from 120 individuals with prediabetes (IFG and/or IGT) at baseline are shown

$0.8 \mathrm{mmol} / \mathrm{l}, p=0.04)$ and $2 \mathrm{~h}(8.6 \pm 1.5 \mathrm{vs} 7.7 \pm 1.6 \mathrm{mmol} / \mathrm{l}$, $p=0.002$ ) glucose levels. As was expected, we found a hyperbolic relationship between insulin sensitivity and insulin secretion (ESM Fig. 1), which allowed us to calculate the $D_{\mathrm{I}}$.

Longitudinal results During the intervention, except for uncorrected insulin secretion, all anthropometric and metabolic variables improved. The largest improvements were found for $\mathrm{D}_{\mathrm{I}}(+$ $34 \%)$, liver fat content $(-34 \%)$ and insulin sensitivity $(+23 \%)$ (ESM Table 1). Nevertheless, 55\% of the participants did not revert to NGR. There was large variability between the changes in body fat mass and $2 \mathrm{~h}$ glucose levels (Fig. 1). Even among participants with the largest amount of body fat loss (upper quartile: $-6.9 \pm 3.3 \%$; decrease in body weight $-5.1 \pm 4.9 \mathrm{~kg}$ ), $2 \mathrm{~h}$ blood glucose levels did not improve in $27 \%$ of these individuals and $40 \%$ did not revert to NGR. We found that the changes in insulin sensitivity correlated with changes in body fat measured by BIA (all 120 participants) and MRT ( $n=103)$, as well as with visceral fat mass and liver fat content $(n=101)$. Again these relationships were weaker or absent between $\mathrm{D}_{\mathrm{I}}$ and fasting and $2 \mathrm{~h}$ glucose levels and these variables (Table 2).

We then investigated whether a characteristic phenotype at baseline associates with the restoration of NGR. Among the variable sex and the continuous baseline variables age, BMI, waist circumference, liver fat content, insulin sensitivity, $2 \mathrm{~h}$ glucose levels, fasting glucose levels, insulin secretion and the interaction insulin sensitivity $\times$ liver fat content, the last three associated with restoration of NGR in a multivariate nominal logistic model (Table 3).

On the basis of the well-established role of insulin secretion and insulin sensitivity for the prediction of diabetes, we first divided the participants into two groups, without accounting for NAFLD: a high-risk phenotype, with low $\mathrm{D}_{\mathrm{I}}$ (lower than the median of the group), and low insulin sensitivity (lower than the median of the group), which is insulin secretion failure in the presence of insulin resistance vs a phenotype with all other traits. The decrease in body weight was similar in both groups (Fig. 2a) as was the decrease in $2 \mathrm{~h}$ glucose levels (improvement in glucose tolerance; Fig. 2b). Importantly, restoration of NGR (reaching the status normal fasting glucose $[\mathrm{NFG}]+$ normal glucose tolerance $[\mathrm{NGT}]$ ) was not statistically significant between these phenotypes ( $p=0.07$ ).

Next, we divided participants into a high-risk phenotype based on low $\mathrm{D}_{\mathrm{I}}(n=60)$ or low insulin sensitivity +NAFLD (insulin secretion failure or metabolically malignant NAFLD [25]; $n=12$ ) vs all other phenotypes (Table 4). For a similar decrease in body weight (Fig. 2c; adjusted decrease in body fat $-5.7 \pm 15.3 \%$ in the high-risk phenotype vs $-7.7 \pm 15.2 \%$ in the low-risk phenotype; $p=0.49$ ), individuals with the high-risk phenotype had a smaller decrease in $2 \mathrm{~h}$ blood glucose levels than those with the low-risk phenotype (Fig. 2 d, $-3.7 \pm 20.3 \%$ in the high-risk vs $-18.5 \pm 20.0 \%$ in the low-risk phenotype, $p=0.0009$ ). Additional adjustment for fat mass at baseline and at follow-up ( $p=0.004)$, antihypertensive medication $(p=0.002)$ or lipid-lowering medication $(p=0.001)$ did not appreciably affect these results. Moreover, $67 \%$ of the individuals with this low-risk phenotype, but only $31 \%$ of the participants with the high-risk phenotype, reached the status NFG+NGT (Table 4). The odds ratio for reaching the status NGR was $4.54(95 \%$ CI $2.08,9.94)$ for participants having the low-risk phenotype. The test had a positive predictive value of $67 \%$ and a negative predictive value of $69 \%$.

Table 2 Relationships of changes of body fat mass and body fat distribution with changes of metabolic variables

\begin{tabular}{|c|c|c|c|c|c|c|c|c|}
\hline \multirow[t]{2}{*}{ Variable } & \multicolumn{2}{|c|}{ Change in body fat ${ }_{\text {BIA }}$} & \multicolumn{2}{|c|}{ Change in body fat ${ }_{\mathrm{MRT}}{ }^{\mathrm{a}}$} & \multicolumn{2}{|c|}{ Change in visceral fat ${ }_{M R T}{ }^{a}$} & \multicolumn{2}{|c|}{ Change in liver fat ${ }_{\mathrm{MRS}}{ }^{\mathrm{b}}$} \\
\hline & $r$ & $p$ & $r$ & $p$ & $r$ & $p$ & $r$ & $p$ \\
\hline Change in insulin sensitivity & -0.29 & 0.001 & -0.33 & 0.0006 & -0.27 & 0.007 & -0.19 & 0.05 \\
\hline Change in the $D_{I}$ & -0.21 & 0.03 & -0.20 & 0.04 & -0.24 & 0.02 & -0.19 & 0.05 \\
\hline Change in fasting glucose levels & 0.12 & 0.19 & 0.31 & 0.002 & 0.32 & 0.001 & 0.10 & 0.31 \\
\hline Change in $2 \mathrm{~h}$ glucose levels & 0.18 & 0.049 & 0.10 & 0.30 & 0.15 & 0.14 & 0.19 & 0.06 \\
\hline
\end{tabular}

Changes (fold-change) during the lifestyle intervention

$r$, Pearson correlation coefficient; MRS, magnetic resonance spectroscopy

${ }^{\mathrm{a}}$ Total and visceral fat mass and ${ }^{\mathrm{b}}$ liver fat content at follow-up were only available in 103 and 101 individuals, respectively, at follow-up 
Table 3 Baseline variables for the prediction of the restoration of NGR in a multivariate nominal logistic model

\begin{tabular}{lll}
\hline Variable & Likelihood-ratio $\chi^{2}$ & $p$ value \\
\hline Sex & 0.184 & 0.67 \\
Age & 0.009 & 0.92 \\
BMI & 0.005 & 0.95 \\
Waist circumference & 1.975 & 0.16 \\
Fasting glucose levels & 4.131 & 0.042 \\
2 h glucose levels & 1.014 & 0.31 \\
Insulin secretion & 6.807 & 0.009 \\
Insulin sensitivity & 1.826 & 0.18 \\
Liver fat content & 1.962 & 0.16 \\
Insulin sensitivity $\times$ liver fat content & 4.766 & 0.029 \\
\hline
\end{tabular}

\section{Discussion}

In the present study, we investigated whether at-risk phenotypes can be identified in individuals with prediabetes at baseline that are associated with a reduced response in the improvement of glycaemia during a lifestyle intervention. We found that a phenotype, consisting of low insulin secretion relative to insulin resistance or insulin-resistant NAFLD, was associated with reduced improvement in glucose
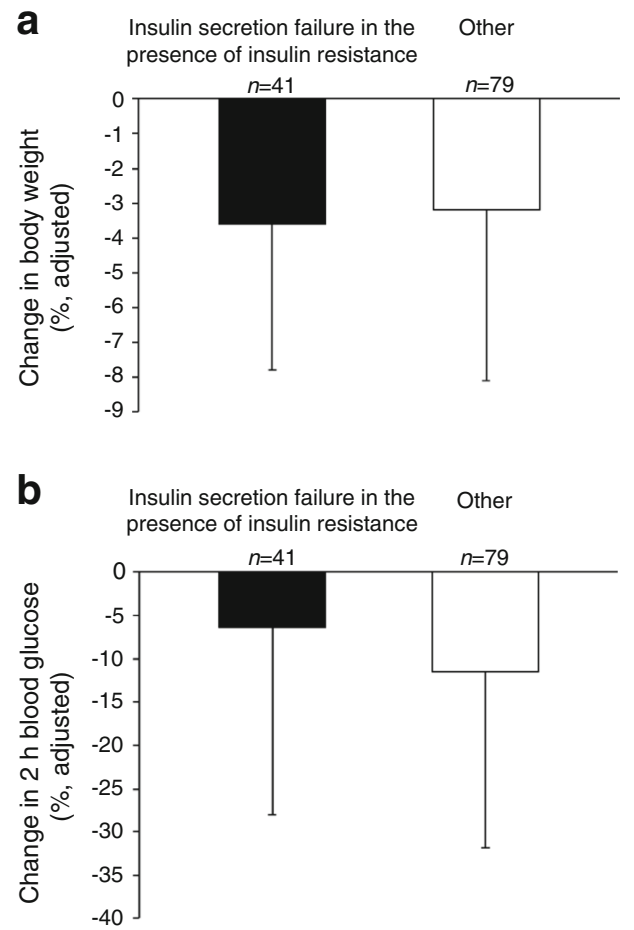

Fig. 2 Change in body weight and glucose tolerance in individuals with high-risk and low-risk phenotypes during lifestyle intervention. A total of 120 individuals with prediabetes (IFG and/or IGT) were retrospectively divided into two high-risk phenotypes based on baseline traits: (1) low $\mathrm{D}_{\mathrm{I}}$ and low insulin sensitivity (insulin secretion failure in the presence of insulin resistance; $\mathbf{a}$ and b); and (2) low $\mathrm{D}_{\mathrm{I}}$ or low insulin sensitivity+ tolerance, and less reversal of prediabetes, during such an intervention. These findings support previous observations that prediabetes is a very heterogeneous condition [26-28]. Moreover, they indicate that the identification of specific prediabetes phenotypes prior to an intervention may help not only to assess the response regarding progression to diabetes but also to implement (early on) more-intensive interventions in individuals with high-risk phenotypes.

We undertook the present analysis based on the observation from clinical lifestyle intervention trials that the improvement of glycaemia, which was achieved during the intervention, was relatively heterogeneous [1-14]. Furthermore, several problems about successful translation of such interventions to clinical practice have been identified [29-31]. Thus, while it would be desirable to implement safe lifestyle intervention programmes in individuals at risk for diabetes nationwide, the relatively high costs and the relatively large variability in the success rates of such an intervention limit this endeavour.

To overcome these problems, it may be necessary to better adapt such a lifestyle intervention towards the individual's risk of diabetes, and more importantly, this adaptation could optimise the chance that the intervention would improve the glycaemia of the individual. Analyses from clinical lifestyle intervention trials have consistently shown that prevention of diabetes is correlated to the amount of weight loss that has
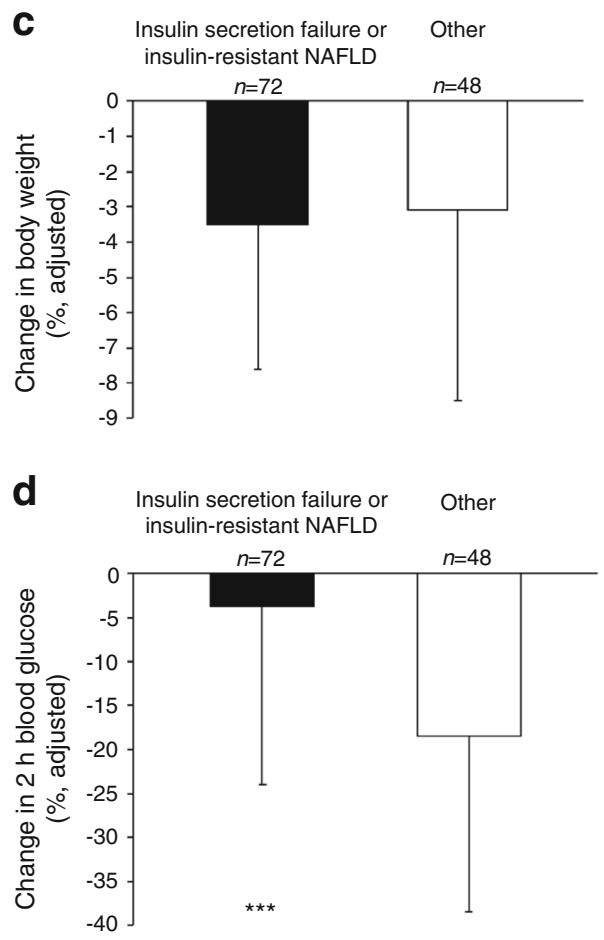

NAFLD (insulin secretion failure or insulin-resistant NAFLD; $\mathbf{c}$ and d). These two high-risk groups were compared with the respective low-risk phenotypes comprising the other traits. Data are least square means and $\mathrm{SD}$ (for differences between the groups in the changes of body weight and $2 \mathrm{~h}$ glucose levels, each adjusted for age, sex and the respective level of the variable at baseline, in multivariate regression models). ${ }^{* * *} p<0.001$ 
Table 4 Selected participant characteristics at baseline and after 9 months of lifestyle intervention in the high-risk and low-risk phenotypes based on insulin secretion, insulin resistance and NAFLD

\begin{tabular}{|c|c|c|c|c|c|c|}
\hline \multirow[t]{2}{*}{ Variable } & \multicolumn{3}{|c|}{$\begin{array}{l}\text { High-risk phenotype (low } \mathrm{D}_{\mathrm{I}} \text { or low insulin } \\
\text { sensitivity +NAFLD; } n=72)^{\mathrm{a}}\end{array}$} & \multicolumn{3}{|c|}{ Low-risk phenotype (all other traits, $n=48)^{\mathrm{b}}$} \\
\hline & Baseline & Follow-up & $p$ value & Baseline & Follow-up & $p$ value \\
\hline IFG/IGT/IFG+IGT/DIA & $15 / 22 / 35 / 0$ & $14 / 16 / 17 / 3$ & & $29 / 17 / 2 / 0$ & $8 / 4 / 4 / 0$ & \\
\hline $\mathrm{NFG}+\mathrm{NGT}[N(\%)]$ & 0 & $22(31)$ & & 0 & $32(67)$ & \\
\hline $\operatorname{BMI}\left(\mathrm{kg} / \mathrm{m}^{2}\right)$ & $30.9 \pm 4.9$ & $29.8 \pm 4.6$ & $<0.0001$ & $28.7 \pm 4.4$ & $27.8 \pm 4.4$ & 0.0006 \\
\hline HPA score & $7.98 \pm 1.1$ & $8.51 \pm 1.0$ & $<0.0001$ & $8.1 \pm 1.0$ & $8.56 \pm 0.8$ & 0.0002 \\
\hline Energy intake $(\mathrm{kJ})$ & $8,307 \pm 2,332$ & $7,611 \pm 1,523$ & 0.001 & $8,038 \pm 1,928$ & $7,647 \pm 1,793$ & 0.04 \\
\hline Fasting blood glucose $(\mathrm{mmol} / \mathrm{l})$ & $5.7 \pm 0.5$ & $5.5 \pm 0.5$ & $<0.0001$ & $5.5 \pm 0.5$ & $5.2 \pm 0.4$ & 0.003 \\
\hline $2 \mathrm{~h}$ blood glucose $(\mathrm{mmol} / \mathrm{l})$ & $9.5 \pm 1.5$ & $8.8 \pm 1.9$ & 0.0005 & $7.6 \pm 1.4$ & $7.0 \pm 1.4$ & 0.017 \\
\hline Insulin sensitivity (AU) & $7.8 \pm 4.6$ & $9.9 \pm 5.4$ & $<0.0001$ & $13.1 \pm 4.5$ & $16.0 \pm 8.4$ & 0.039 \\
\hline IGI (AU) & $109 \pm 79$ & $111 \pm 76$ & 0.13 & $114 \pm 55$ & $130 \pm 93$ & 0.28 \\
\hline $\mathrm{D}_{\mathrm{I}}$ & $649 \pm 318$ & $879 \pm 475$ & $<0.0001$ & $1,361 \pm 599$ & $1,878 \pm 1,728$ & 0.016 \\
\hline Liver fat content $(\%)^{c}$ & $10.9 \pm 8.6$ & $6.6 \pm 5.6$ & $<0.0001$ & $4.4 \pm 4.5$ & $3.9 \pm 3.8$ & 0.21 \\
\hline Low $\mathrm{D}_{\mathrm{I}}(\%)$ & 83 & & & 0 & & \\
\hline Low insulin sensitivity (\%) & 74 & & & 15 & & \\
\hline NAFLD (\%) & 61 & & & 21 & & \\
\hline
\end{tabular}

Data are means $\pm \mathrm{SD}$

${ }^{\text {a }}$ Male/female (40/32) and age $51 \pm 10$ years

${ }^{\mathrm{b}}$ Male/female (31/17) and age $47 \pm 11$ years

${ }^{\mathrm{c}}$ Liver fat content at follow-up was only available in 101 individuals

DIA, diabetes; IGI, insulinogenic index

been achieved in the studies [13, 32]. However, in contrast to the relatively strong correlation between change in weight loss and insulin sensitivity, the correlations of changes of weight loss or fat mass with changes of glycaemia are not very strong, a finding that we have replicated in our present analysis. Furthermore, paradoxically, increased weight loss was associated with increased diabetes incidence in DPPOS, independently of the treatment in the Diabetes Prevention Program (DPP) [12]. Thus, what other variables, preferably those which can be measured prior to the intervention, can help to estimate the effectiveness of the lifestyle intervention to improve glucose levels? So far, low insulin secretion was found to be a good predictor in the Finnish DPS and the U.S. DPP $[11,13]$. Interestingly, in these studies, estimates of insulin resistance were not or were only weakly associated with the improvement of glycaemia.

Consequently, we hypothesised that an at-risk phenotype consisting of low insulin secretion relative to insulin resistance and low insulin sensitivity (as defined by the median values in our population with prediabetes) may be associated with reduced effectiveness of lifestyle intervention to improve $2 \mathrm{~h}$ glucose levels and with the conversion to NGR. For a very similar amount of weight loss, decrease in energy intake and increase in HPA (indicating similar adherence to the intervention), individuals with this phenotype had a lower decrease in
$2 \mathrm{~h}$ glucose levels than those without this phenotype; however, this difference was not statistically significant (Fig. 2a,b).

On the basis of the role of NAFLD in the pathogenesis of metabolic diseases [33-37], we then hypothesised that accounting specifically for insulin resistance+NAFLD (metabolically malignant fatty liver) [25] may help to better estimate such a response. Indeed, for an almost identical weight loss compared with the respective low-risk phenotype, individuals with this at-risk phenotype had only a relative decrease of $2 \mathrm{~h}$ glucose levels of $3.7 \pm 20.3 \%$ while the decrease of glucose amounted to $18.5 \pm 20.0 \%$ in the low-risk phenotype (Fig. 2c,d). Furthermore, only half of the participants with the at-risk phenotype reverted to the status of NGR.

What are the limitations and the strengths of our study? Owing to the fact that the phenotypes isolated IFG, isolated IGT and IFG and IGT combined somewhat differ in their pathophysiology, [26-28] it would be important to know whether the risk phenotypes are associated with the response to the intervention to a similar extent in these prediabetes subphenotypes. However, our sample size was too small to satisfactorily address this question in our study. Nevertheless, in a statistical model, isolated IFG, isolated IGT and IFG and IGT combined did not interact with the risk phenotypes in determining change in glycaemia (e.g. the $p$ value for interaction on change in $2 \mathrm{~h}$ glycaemia was 0.79 ), indicating that the 
results are similar in the three prediabetes subphenotypes. Furthermore, it is not clear whether the findings are similar among different ethnic groups.

It is encouraging that precise phenotyping of individuals with prediabetes prior to the lifestyle intervention may help to better assess the response in respect to the improvement of glycaemia during the intervention. However, two questions arise. First, is it necessary to perform more intense phenotyping, such as a frequently sampled OGTT and to diagnose NAFLD, in order to better estimate the risk of reduced response to the intervention and what value is there in using changes in anthropometric and metabolic variables during the intervention for this purpose? Second, how can such phenotype setting be implemented in daily clinical practice? In our study, insulin secretion and insulin sensitivity (both measured during the OGTT at baseline) and the interaction of insulin sensitivity with NAFLD (also measured at baseline) were more strongly associated with the restoration of NGR than the other variables tested. Fasting and $2 \mathrm{~h}$ glucose levels were not identified as strong determinants. In addition, we focused only on baseline variables because they can help to estimate the response to the intervention already prior to the start of an intervention programme. Thus, based on our main hypothesis, rather than create a complex formula by using continuous and categorical variables, it may be possible to use the variables low insulin secretion and low insulin sensitivity in combination with NAFLD to identify specific pathophysiology-based phenotypes that are associated with the restoration of NGR. In addition, a 2 h OGTT with measurements of stimulated glucose and insulin levels during fasting and at two additional time points already provides reliable information that can be used to calculate insulin secretion and insulin sensitivity. Furthermore, while ultrasonography is inferior to the ${ }^{1} \mathrm{H}$-MRS method to precisely quantify liver fat content, it can be routinely used for the diagnosis of NAFLD [38].

The next questions are, can a reduced response to a standard lifestyle intervention in individuals with the identified high-risk phenotype be overcome by an intensified lifestyle intervention? In all of our participants with prediabetes, change in body fat mass was not associated with the change in $2 \mathrm{~h}$ glucose levels $(p=0.15)$; however, in the high-risk group, a larger decrease of body fat mass correlated with a larger decrease of $2 \mathrm{~h}$ glucose levels ( $p=0.04$, data no shown). Although these findings do not prove that a more intensified intervention is more effective in the high-risk group, they support that it is worth testing this hypothesis in a future study. Furthermore, individuals with the low-risk phenotype may not necessarily require lifestyle intervention, as information about a healthy lifestyle may be sufficient to make them change their lifestyle to prevent progression to diabetes. These questions can only be answered by a randomised and controlled clinical trial. Consequently, we have started the German Prediabetes
Lifestyle Intervention Study (PLIS) in eight centres throughout Germany. A total of 1,000 individuals with prediabetes are being phenotyped for insulin secretion, insulin sensitivity and NAFLD at baseline. Subsequently, participants with the highrisk phenotype are being randomly assigned to a standard and an intensified lifestyle intervention, while individuals with the low-risk phenotype are being randomly assigned to a standard intervention or no intervention (ClinicalTrials.gov, NCT01947595).

In conclusion, we found that stratification of individuals with prediabetes at baseline into a high-risk and a low-risk phenotype, based on corrected insulin secretion and insulinresistant NAFLD, may help to assess the effectiveness of a lifestyle intervention to revert individuals to NGR. This phenotyping approach may help not only to estimate the effectiveness of a lifestyle intervention to prevent type 2 diabetes but also to provide a more intensified intervention to the people with the highest risk for diabetes.

Funding The study was supported in part by grants from the Deutsche Forschungsgemeinschaft (KFO 114), the German Federal Ministry of Education and Research (BMBF) to the German Centre for Diabetes Research (DZD) and iMed - the Helmholtz Initiative on Personalized Medicine. NS was supported by a Heisenberg Professorship from the Deutsche Forschungsgemeinschaft (STE 1096/3-1).

Duality of interest The authors declare that there is no duality of interest associated with this manuscript.

Contribution statement All authors provided substantial contributions to conception and design, acquisition of data or analysis and interpretation of data, drafting the article or revising it critically for important intellectual content and final approval of the version to be published. The most important contributions of each author were as follows. NS and AF researched data and wrote the manuscript. HS, RW, JM and FS researched data and edited the manuscript. NS, AF and HUH designed the study, contributed to the discussion and reviewed the manuscript. NS is the guarantor of this work and, as such, had full access to all the data in the study and takes responsibility for the integrity of the data and the accuracy of the data analysis.

\section{References}

1. Tuomilehto J, Lindström J, Eriksson JG et al (2001) Prevention of type 2 diabetes mellitus by changes in lifestyle among subjects with impaired glucose tolerance. N Engl J Med 344:1343-1350

2. Lindström J, Ilanne-Parikka P, Peltonen M et al (2006) Sustained reduction in the incidence of type 2 diabetes by lifestyle intervention: follow-up of the Finnish Diabetes Prevention Study. Lancet 368:1673-1679

3. Knowler WC, Barrett-Connor E, Fowler SE et al (2002) Reduction in the incidence of type 2 diabetes with lifestyle intervention or metformin. N Engl J Med 346:393-403

4. Diabetes Prevention Program Research Group, Knowler WC, Fowler SE et al (2009) 10-year follow-up of diabetes incidence and weight loss in the Diabetes Prevention Program Outcomes Study. Lancet 374:1677-1686 
5. Pan XR, Li GW, Hu YH et al (1997) Effects of diet and exercise in preventing NIDDM in people with impaired glucose tolerance. The Da Qing IGT and Diabetes Study. Diabetes Care 20:537-544

6. Li G, Zhang P, Wang J et al (2008) The long-term effect of lifestyle interventions to prevent diabetes in the China Da Qing Diabetes Prevention Study: a 20-year follow-up study. Lancet 371:1783-1789

7. Lindström J1, Peltonen M, Eriksson JG et al (2013) Improved lifestyle and decreased diabetes risk over 13 years: long-term followup of the randomised Finnish Diabetes Prevention Study (DPS). Diabetologia 56:284-293

8. Schwarz PE, Greaves CJ, Lindström J, Yates T, Davies MJ (2012) Nonpharmacological interventions for the prevention of type 2 diabetes mellitus. Nat Rev Endocrinol 8:363-373

9. Ramachandran A, Snehalatha C, Ram J et al (2013) Effectiveness of mobile phone messaging in prevention of type 2 diabetes by lifestyle modification in men in India: a prospective, parallel-group, randomised controlled trial. Lancet Diabetes Endocrinol 1:191-198

10. Glechner A, Harreiter J, Gartlehner G et al (2015) Sex-specific differences in diabetes prevention: a systematic review and metaanalysis. Diabetologia 58:242-254

11. Lindström J1, Peltonen M, Eriksson JG et al (2008) Determinants for the effectiveness of lifestyle intervention in the Finnish Diabetes Prevention Study. Diabetes Care 31:857-862

12. Perreault L, Pan Q, Mather KJ et al (2012) Effect of regression from prediabetes to normal glucose regulation on long-term reduction in diabetes risk: results from the Diabetes Prevention Program Outcomes Study. Lancet 379:2243-2251

13. Perreault L, Kahn SE, Christophi CA, Knowler WC, Hamman RF, Diabetes Prevention Program Research Group (2009) Regression from pre-diabetes to normal glucose regulation in the diabetes prevention program. Diabetes Care 32:1583-1588

14. Kantartzis K, Thamer C, Peter A et al (2009) High cardiorespiratory fitness is an independent predictor of the reduction in liver fat during a lifestyle intervention in non-alcoholic fatty liver disease. Gut 58:1281-1288

15. Kantartzis K, Machann J, Schick F et al (2011) Effects of a lifestyle intervention in metabolically benign and malign obesity. Diabetologia 54:864-868

16. Lehmann R1, Franken H, Dammeier S et al (2013) Circulating lysophosphatidylcholines are markers of a metabolically benign nonalcoholic fatty liver. Diabetes Care 36:2331-2338

17. Stefan N, Thamer C, Staiger H et al (2007) Genetic variations in PPARD and PPARGC1A determine mitochondrial function and change in aerobic physical fitness and insulin sensitivity during lifestyle intervention. J Clin Endocrinol Metab 92:1827-1833

18. Baecke JA, Burema J, Frijters JE (1982) A short questionnaire for the measurement of habitual physical activity in epidemiological studies. Am J Clin Nutr 36:936-942

19. Machann J, Thamer C, Stefan N et al (2010) Follow-up whole-body assessment of adipose tissue compartments during a lifestyle intervention in a large cohort at increased risk for type 2 diabetes. Radiology 257:353-363

20. Szczepaniak LS, Nurenberg P, Leonard D et al (2005) Magnetic resonance spectroscopy to measure hepatic triglyceride content: prevalence of hepatic steatosis in the general population. Am J Physiol Endocrinol Metab 288:E462-468

21. Genuth S, Alberti KG, Bennett P et al (2003) Expert Committee on the Diagnosis and Classification of Diabetes Mellitus. Follow-up report on the diagnosis of diabetes mellitus. Diabetes Care 26:3160-3167

22. Matsuda M, DeFronzo RA (1999) Insulin sensitivity indices obtained from oral glucose tolerance testing: comparison with the euglycemic insulin clamp. Diabetes Care 22:1462-1470

23. Thamer C, Machann J, Stefan $\mathrm{N}$ et al (2007) High visceral fat mass and high liver fat are associated with resistance to lifestyle intervention. Obesity 15:531-538

24. Nanditha A, Ram J, Snehalatha C et al (2014) Early improvement predicts reduced risk of incident diabetes and improved cardiovascular risk in prediabetic Asian Indian men participating in a 2-year lifestyle intervention program. Diabetes Care 37:3009-3015

25. Stefan N, Häring HU (2011) The metabolically benign and malignant fatty liver. Diabetes 60:2011-2017

26. Stancáková A, Javorský M, Kuulasmaa T, Haffner SM, Kuusisto J, Laakso M (2009) Changes in insulin sensitivity and insulin release in relation to glycemia and glucose tolerance in 6,414 Finnish men. Diabetes 58:1212-1221

27. Tabák AG, Herder C, Rathmann W, Brunner EJ, Kivimäki M (2012) Prediabetes: a high-risk state for diabetes development. Lancet 379:2279-2290

28. Kahn SE, Cooper ME, Del Prato S (2014) Pathophysiology and treatment of type 2 diabetes: perspectives on the past, present, and future. Lancet 383:1068-1083

29. Cefalu WT (2014) A "spoonful of sugar" and the realities of diabetes prevention! Diabetes Care 37:906-908

30. Dunkley AJ, Bodicoat DH, Greaves CJ et al (2014) Diabetes prevention in the real world: effectiveness of pragmatic lifestyle interventions for the prevention of type 2 diabetes and of the impact of adherence to guideline recommendations: a systematic review and meta-analysis. Diabetes Care 37:922-933

31. Kahn R, Davidson MB (2014) The reality of type 2 diabetes prevention. Diabetes Care 37:943-949

32. Uusitupa M, Lindi V, Louheranta A et al (2003) Long-term improvement in insulin sensitivity by changing lifestyles of people with impaired glucose tolerance: 4-year results from the Finnish Diabetes Prevention Study. Diabetes 52:2532-2538

33. Gastaldelli A, Kozakova M, Højlund K et al (2009) Fatty liver is associated with insulin resistance, risk of coronary heart disease, and early atherosclerosis in a large European population. Hepatology 49:1537-1544

34. Finucane FM, Sharp SJ, Hatunic M et al (2014) Liver fat accumulation is associated with reduced hepatic insulin extraction and beta cell dysfunction in healthy older individuals. Diabetol Metab Syndr 6:43

35. Roden M (2006) Mechanisms of disease: hepatic steatosis in type 2 diabetes - pathogenesis and clinical relevance. Nat Clin Pract Endocrinol Metab 2:335-348

36. Cusi K (2012) Role of obesity and lipotoxicity in the development of nonalcoholic steatohepatitis: pathophysiology and clinical implications. Gastroenterology 142:711-725.e6

37. Stefan N, Häring HU (2013) The role of hepatokines in metabolism. Nat Rev Endocrinol 9:144-152

38. Schwenzer NF, Springer F, Schraml C, Stefan N, Machann J, Schick F (2009) Non-invasive assessment and quantification of liver steatosis by ultrasound, computed tomography and magnetic resonance. J Hepatol 51:433-445 